\title{
SPECTRAL TRANSFORMATIONS AND GENERALIZED POLLACZEK POLYNOMIALS*
}

\author{
OKSANA YERMOLAYEVA ${ }^{\dagger}$ AND ALEXEI ZHEDANOV ${ }^{\ddagger}$
}

To Richard Askey on his 65th birthday

\begin{abstract}
The Christoffel and the Geronimus transformations of the classical orthogonal polynomials of a discrete variable are exploited to construct new families of the generalized Pollaczek polynomials. The recurrence coefficients $u_{n}, b_{n}$ of these polynomials are rational functions of the argument $n$. The (positive) weight function is known explicitly. These polynomials are shown to belong to a subclass of the semi-classical orthogonal polynomials of a discrete variable. We illustrate the method by constructing a family of the modified Charlier polynomials which are orthogonal with respect to a perturbed Poisson distribution. The generating function of these polynomials provides a nontrivial extension of the class of the Meixner-Sheffer generating functions.
\end{abstract}

1. Introduction. The formal orthogonal polynomials $P_{n}(x)$ are defined through the three-term recurrence relation ([8])

$$
P_{n+1}(x)+u_{n} P_{n-1}(x)+b_{n} P_{n}(x)=x P_{n}(x)
$$

with the initial conditions

$$
P_{0}(x)=1, \quad P_{1}(x)=x-b_{0} .
$$

The polynomials $P_{n}(x)$ are monic (i.e. $P_{n}(x)=x^{n}+O\left(x^{n-1}\right)$ ). It can be shown that for arbitrary complex coefficients $b_{n}$ and (non-zero) $u_{n}$ there exists a linear functional $\mathcal{L}$ such that

$$
\mathcal{L}\left\{P_{n}(x) x^{k}\right\}=h_{n} \delta_{n k}, k \leq n,
$$

where

$$
h_{n}=u_{1} u_{2} \cdots u_{n} \neq 0
$$

are normalization constants.

The functional $\mathcal{L}$ is defined on the space of polynomials by its moments

$$
c_{n}=\mathcal{L}\left\{x^{n}\right\}, n=0,1, \cdots .
$$

The standard normalization condition is $h_{0}=c_{0}=1$.

The condition (1.3) can be rewritten in the form of the orthogonality relation [8]

$$
\mathcal{L}\left\{P_{n}(x) P_{m}(x)\right\}=h_{n} \delta_{n m}, n, m=0,1, \cdots .
$$

If, in addition, the coefficients $b_{n}$ are real and $u_{n}>0$ then there exists a positive Borel measure $\mu$ on the real line such that

$$
c_{n}=\int_{-\infty}^{\infty} x^{n} d \mu(x)
$$

\footnotetext{
* Received Jan. 14, 1998, revised May 15, 1998.

${ }^{\dagger}$ Department of Mathematics and Statistics, Concordia University, 7141 Sherbrooke str. West, Montreal, Quebec H4B 1R6, Canada (oksana@neumann.concordia.ca).

$\ddagger$ Donetsk Institute for Physics and Technology, Donetsk 340114, Ukraine (zhedanov@kinetic. ac.donetsk.ua).
} 
and hence the orthogonality condition can be presented in the form (the so-called Favard theorem [8])

$$
\int_{-\infty}^{\infty} P_{n}(x) P_{m}(x) d \mu(x)=h_{n} \delta_{n m} .
$$

Sometimes it is convenient to deal with the weight function $w(x)$ defined by $d \mu(x)=$ $w(x) d x$. In the case of a purely discrete measure the weight function can be written in terms of Dirac delta functions $w(x)=\sum_{k} M_{k} \delta\left(x-x_{k}\right)$, where the discrete masses $M_{k}$ are located at the spectral points $x_{k}$.

The Stieltjes function $F(z)$ is defined as [8]

$$
F(z)=\mathcal{L}\left\{(z-x)^{-1}\right\},
$$

where the functional $\mathcal{L}$ acts upon the $x$ variable. The Stieltjes function is the generating function of the moments

$$
F(z)=\sum_{k=0}^{\infty} \frac{c_{k}}{z^{k+1}}
$$

On the other hand there is an important expansion in terms of the continued fraction $([1])$

$$
F(x)=\frac{1}{x-b_{0}-\frac{u_{1}}{x-b_{1}-\frac{u_{2}}{x-b_{2}-\cdots}}} .
$$

Formula (1.11) allows one to find (in principle) the Stieltjes function $F(z)$ from the given recurrence coefficients $u_{n}, b_{n}$. The measure $\mu$ can then be restored by means of the inverse Stieltjes transform ([1]). Obviously such procedure is rather implicit (one should evaluate infinite continuous fraction (1.11)).

Hence it is desirable to have a list of "nice" orthogonal polynomials such that both the measure and the recurrence coefficients are known explicitly.

We will call that OP belong to the generalized Pollaczek class (GPC) if their recurrence coefficients $u_{n}, b_{n}$ are rational functions in $n$ whereas the weight function $w(x)$ is known explicitly.

Note that Pollaczek [23] proposed to study a class of OP such that the recurrence coefficients are rational functions in $n$ with the additional restrictions

$$
\lim _{n \rightarrow \infty} u_{n}=\text { const, } \lim _{n \rightarrow \infty} b_{n}=\text { const. }
$$

These restrictions are quite natural, for they lead to OP generalizing the Jacobi polynomials with the measure located on a finite interval of the real axis.

Pollaczek stressed that such polynomials are important because their generating function satisfies some linear differential equation (of the order $\geq 2$ ), hence some properties of these polynomials can be restored from the behavior of solutions of the corresponding differential equation.

Our definition of GPC is slightly less restrictive: we do not impose asymptotic conditions upon the recurrence coefficients, but on the other hand we demand that the weight function should be known explicitly. 
The following OP belonging to GPC are known today:

1) the classical Jacobi polynomials and their specializations (i.e. the Laguerre and Hermite polynomials);

2) "non-classical" the Krall's polynomials satisfying a higher-order linear differential eigenvalue equation (the so-called polynomials of the "Jacobi-type", "Legendretype" etc. , see, e.g. [18], [17]). The measure of these polynomials is obtained from the measures of the classical Jacobi and Laguerre polynomials by adding of one or two discrete masses at the endpoints of the orthogonality interval. Koornwider [15] considered the polynomials obtained by inserting of two arbitrary masses at the endpoints of the orthogonality interval of the arbitrary Jacobi polynomials. Some classes of the Koornwinder's polynomials also belong to GPC. In all these cases restrictions (1.12) are fulfilled.

3) the Wilson polynomials [32] and all their specializations: Hahn, Meixner, Pollaczek, Krawtchouk etc. - see [14] for a full list. In this case, instead of Pollaczek restriction (1.12), we have

$$
u_{n} \propto n^{4}, b_{n} \propto n^{2}
$$

for the Wilson and continuous dual Hahn polynomials;

$$
u_{n} \propto n^{2}, b_{n} \propto n,
$$

for the continuous Hahn, Meixner and Pollaczek polynomials and

$$
u_{n} \propto n, b_{n} \propto n
$$

for the Charlier polynomials.

4)the so-called Random Walk Polynomials studied in [3]. For these polynomials the Pollaczek restriction (1.12) is fulfilled and the spectrum contains both continuous and discrete parts. These polynomials admit a generalization studied in [7].

5)OP connected with the elliptic functions [8]. There are several examples. For one of them $u_{n}=\kappa^{2}(2 n)^{2}\left(4 n^{2}-1\right), b_{n}=\left(\kappa^{2}+1\right)(2 n+1)^{2}$, where $0<\kappa<1$. The orthogonality measure of these polynomials (found by Stieltjes) is purely discrete one. For other OP related with this example see, e.g. [30].

The main goal of this paper is to present a new class of GPC polynomials which are a generalization of the above class 3 ). We construct infinitely many such OP with somewhat unusual spectral property. Namely, all these OP have a purely discrete spectrum. This spectrum is obtained from the spectrum of the classical OP of a discrete variable by cancelling of some spectral points.

In the next section we give a brief introduction to theory of spectral transformations of OP. These transformations play an important role in studying of the classical and semi-classical OP (see also [25], [34]).

2. Spectral transformations of the orthogonal polynomials. In this section we describe spectral transformations of the orthogonal polynomials on the interval. These transformations were introduced by Christoffel (1858) and Geronimus (1940).

The Christoffel transform of OP is defined by the formula [29]

$$
\tilde{P}_{n}(x)=\frac{P_{n+1}(x)-A_{n} P_{n}(x)}{x-\alpha}
$$


where $A_{n}=P_{n+1}(\alpha) / P_{n}(\alpha)$ and $\alpha$ is an arbitrary parameter which does not coincide with the roots of the polynomials $P_{n}(x)$.

The weight function $w(x)$ and the Stieltjes functions $F(x)$ are transformed as

$$
\begin{aligned}
& \tilde{w}(x)=\frac{(x-\alpha) w(x)}{c_{1}-\alpha}, \\
& \tilde{F}(x)=\frac{(x-\alpha) F(x)-1}{c_{1}-\alpha} .
\end{aligned}
$$

It is easily seen that the condition $\tilde{w}(x)>0$ leads to the restriction $\alpha \leq a_{1}$ or $\alpha \geq a_{2}$, i.e. the auxiliary spectral parameter $\alpha$ should be outside the spectral interval. This restriction is evident from theory of the kernel polynomials [8].

Consider a chain of $N$ CT with the parameters $\alpha_{1}, \alpha_{2}, \cdots, \alpha_{N}$. For the transformed recurrence coefficients we have

$$
\begin{aligned}
u_{n}^{(N)} & =u_{n} \frac{D_{n+1} D_{n-1}}{D_{n}^{2}}, \\
b_{n}^{(N)} & =b_{n+N}+\frac{V_{n+1}}{D_{n+1}}-\frac{V_{n}}{D_{n}},
\end{aligned}
$$

where $D_{n}, V_{n}$ are $N \times N$ determinants defined as

$$
D_{n}=\left|\begin{array}{cccc}
P_{n+N-1}\left(\alpha_{1}\right) & P_{n+N-2}\left(\alpha_{1}\right) & \cdots & P_{n}\left(\alpha_{1}\right) \\
P_{n+N-1}\left(\alpha_{2}\right) & P_{n+N-2}\left(\alpha_{2}\right) & \cdots & P_{n}\left(\alpha_{2}\right) \\
\cdots & \cdots & \cdots & \cdots \\
P_{n+N-1}\left(\alpha_{N}\right) & P_{n+N-2}\left(\alpha_{N}\right) & \cdots & P_{n}\left(\alpha_{N}\right)
\end{array}\right|
$$

and

$$
V_{n}=\left|\begin{array}{cccc}
P_{n+N}\left(\alpha_{1}\right) & P_{n+N-2}\left(\alpha_{1}\right) & \cdots & P_{n}\left(\alpha_{1}\right) \\
P_{n+N}\left(\alpha_{2}\right) & P_{n+N-2}\left(\alpha_{2}\right) & \cdots & P_{n}\left(\alpha_{2}\right) \\
\cdots & \cdots & \cdots & \cdots \\
P_{n+N}\left(\alpha_{N}\right) & P_{n+N-2}\left(\alpha_{N}\right) & \cdots & P_{n}\left(\alpha_{N}\right)
\end{array}\right| .
$$

Geronimus transform GT was found in 1940 [11], [12] (see also [21] and [9], [13] where the similar problem was considered). GT is written as

$$
\tilde{P}_{0}=1, \quad \tilde{P}_{n}(x)=P_{n}(x)-B_{n} P_{n-1}(x), \quad n=1,2, \cdots
$$

where

$$
B_{n}=\phi_{n} / \phi_{n-1}
$$

and the function $\phi_{n}$ is a solution of the recurrence relation (1.1) with auxiliary spectral parameter $\alpha$ :

$$
\phi_{n}=F_{n}(\alpha)+\chi P_{n}(\alpha), \quad n=0,1, \cdots
$$

The parameter $\chi$ is arbitrary, however there are some restrictions needed to provide positivity of the weight function $\tilde{w}(x)$ of the polynomials $\tilde{P}_{n}(x)$ (obviously the restrictions for $\alpha$ are the same as in the case of $\mathrm{CT}$ ). 
The recurrence coefficients are transformed according to the formulas

$$
\begin{array}{lll}
\tilde{u}_{1}=\frac{\phi_{1}}{\phi_{0}}\left(\alpha-b_{0}-\frac{\phi_{1}}{\phi_{0}}\right), & \tilde{u}_{n}=\frac{u_{n-1} B_{n}}{B_{n-1}}, & n=2,3, \cdots, \\
\tilde{b}_{0}=b_{0}+\frac{\phi_{1}}{\phi_{0}}, & \tilde{b}_{n}=b_{n}+B_{n+1}-B_{n}, & n=1,2, \cdots .
\end{array}
$$

For the transformed weight function and the Stieltjes functions one has [11]

$$
\begin{gathered}
\tilde{w}(x)=\frac{\chi \delta(x-\alpha)+w(x)(\alpha-x)^{-1}}{\chi+F(\alpha)} \\
\tilde{F}(x)=\frac{F(\alpha)+\chi-F(x)}{(x-\alpha)(\chi+F(\alpha))}
\end{gathered}
$$

It is easily seen that the Geronimus transform (2.12) is reciprocal to (2.3). However, in contrast to $\mathrm{CT}$ the transform (2.12) contains two free parameters $\alpha$ and $\chi$. The second free parameter $\chi$ determines the value of an additional discrete mass inserted to the spectrum of the polynomials $\tilde{P}_{n}(x)$ as is seen from $(2.11)$.

For the transformed recurrence coefficients under $N$ Geronimus transforms we have

$$
\begin{aligned}
& u_{n}^{(N)}=u_{n-N} \frac{W_{n+1} W_{n-1}}{W_{n}^{2}} \\
& b_{n}^{(N)}=b_{n}+\frac{U_{n+1}}{W_{n+1}}-\frac{U_{n}}{W_{n}}
\end{aligned}
$$

where the $N \times N$ determinants are defined as

$$
W_{n}=\left|\begin{array}{cccc}
\phi_{n-1}\left(\alpha_{1}\right) & \phi_{n-2}\left(\alpha_{1}\right) & \cdots & \phi_{n-N}\left(\alpha_{1}\right) \\
\phi_{n-1}\left(\alpha_{2}\right) & \phi_{n-2}\left(\alpha_{2}\right) & \cdots & \phi_{n-N}\left(\alpha_{2}\right) \\
\cdots & \cdots & \cdots & \cdots \\
\phi_{n-1}\left(\alpha_{N}\right) & \phi_{n-2}\left(\alpha_{N}\right) & \cdots & \phi_{n-N}\left(\alpha_{N}\right)
\end{array}\right|
$$

and

$$
U_{n}=\left|\begin{array}{cccc}
\phi_{n}\left(\alpha_{1}\right) & \phi_{n-2}\left(\alpha_{1}\right) & \cdots & \phi_{n-N}\left(\alpha_{1}\right) \\
\phi_{n}\left(\alpha_{2}\right) & \phi_{n-2}\left(\alpha_{2}\right) & \cdots & \phi_{n-N}\left(\alpha_{2}\right) \\
\cdots & \cdots & \cdots & \cdots \\
\phi_{n}\left(\alpha_{N}\right) & \phi_{n-2}\left(\alpha_{N}\right) & \cdots & \phi_{n-N}\left(\alpha_{N}\right)
\end{array}\right| .
$$

Note that strictly speaking the formulas (2.13) are valid for $n>N$. For $0 \leq n<$ $N$ one should reconsider these formulas. We will not write down these (auxiliary) formulas here.

3. The Christoffel transforms preserving the positivity property of the weight function. Assume that the weight function $w^{(N)}(x)$ (obtained from $w(x)$ after $N$ CT) preserves the positivity property. For the single Christoffel transform (i.e. $N=1$ ) it is necessary that the auxiliary parameter $\alpha$ lies beyond the orthogonality interval. There is a situation, however, when one can perform arbitrary many CT with the parameter $\alpha_{i}$ chosen inside the orthogonality interval such that the weight function $w^{(N)}(x)$ is still positive. 
Assume that the spectrum of OP is discrete one and bounded from the below:

$$
w(x)=\sum_{i=0}^{N} M_{i} \delta\left(x-x_{i}\right)
$$

where $x_{i}$ are locations of the jumps and $M_{i}>0$ are corresponding concentrated masses. We assume that

$$
x_{i+1}>x_{i}, \quad i=0,1, \cdots
$$

Choose $K$ parameters $\alpha_{i}, i=1,2, \cdots, K$ coinciding with a subset of spectral points, i.e. $\alpha_{i}=x_{\sigma(i)}$ where $\sigma(i)$ is an increasing function of the integer argument $i=$ $0,1,2, \cdots$ taking natural integers.

For the transformed weight function we thus have

$$
w^{(K)}(x)=\sum_{i=0}^{N} M_{i}^{(K)} \delta\left(x-x_{i}\right)
$$

where the transformed masses are

$$
M_{i}^{(K)}=\kappa M_{i}\left(\alpha_{1}-x_{i}\right)\left(\alpha_{2}-x_{i}\right) \cdots\left(\alpha_{K}-x_{i}\right)
$$

Obviously some masses vanish in the sum (3.3): $M_{i}^{(K)}=0$ iff $\alpha_{j}=x_{i}$ for at least one $1 \leq j \leq K$.

The transformed weight function $\tilde{w}(x)$ is positive iff all the rest masses $M_{i}^{(K)}, i=$ $0,1, \cdots, N$ are positive. Thus we need the condition $\left(\alpha_{1}-x_{i}\right)\left(\alpha_{2}-x_{i}\right) \cdots\left(\alpha_{K}-x_{i}\right)>0$ for $i=0,1, \cdots, N$ (excepting those for which $M_{i}^{(K)}=0$ ). Taking into account that all $x_{i}$ are monotonic with respect to $i$ we find that the criterion of the positivity can be formulated in the following manner.

Divide all indices $k$ of $\alpha_{k}$ into groups of "nearest neighbors" $\{k\}=\cup_{m=1}^{L} Y_{m}\left(r_{m}\right)$, where $Y_{m}\left(r_{m}\right)$ is a group joining $r_{m}$ indices going without gaps between them: $Y_{m}\left(r_{m}\right)$ $=\left\{k^{(m)}, k^{(m)}+1, k^{(m)}+2, \cdots, k^{(m)}+r_{m}-1\right\}$, and $m$ is a label of this group $(L$ is the number of all these groups). Obviously $K=r_{1}+r_{2}+\cdots+r_{L}$. We assume a natural ordering for these groups $k_{(m+1)}>k^{(m)}+r_{m}$. The numbers $k^{(m)}$ denote the starting numbers inside each group. If $k^{(1)}=0$ then the first $r_{1}$ spectral parameters $\alpha_{k}$ coincide with the first $r_{1}$ spectral points $x_{0}, x_{1}, \cdots, x_{r_{1}}$.

We will call that the set of indices $\{k\}$ (divided into the groups $Y_{m}\left(r_{m}\right)$ ) is admissible if the following conditions hold:

(i) if $k^{(1)} \neq 0$ then all numbers $r_{m}, m=1,2, \cdots, L$ are even;

(ii) if $k^{(1)}=0$ then the number $r_{1}$ may be arbitrary whereas the rest numbers $r_{m}$ are even.

For example the set of indices $\{k\}=\{0,1,2,7,8,9,10,15,16\}$ is admissible because it contains 3 groups with $r_{1}=3\left(k_{1}=0\right), r_{2}=2\left(k_{2}=7\right), r_{3}=2\left(k_{3}=15\right)$. On the other hand the set $\{k\}=1,2,3,4,7,8,9,13,14$ is not admissible because it contains 3 groups with $r_{1}=4, r_{2}=3, r_{3}=2$, and $r_{2}$ is odd which is forbidden.

Now we can formulate the following

Proposition 1. Assume that some OP $P_{n}(x)$ have a purely discrete positive weight function with the masses $M_{k}>0$ located at the points $x_{k}, k=0,1, \cdots, N \leq \infty$ such that $x_{k+1}>x_{k}$. Assume that $O P P_{n}^{(K)}(x)$ are obtained from $P_{n}(x)$ by application 
of $K$ Christoffel transforms taking at the points $\alpha_{i}, i=1,2 \cdots, K$ coinciding with some subset of $x_{k}$. Then $O P P_{n}^{(K)}(x)$ have positive discrete weight function iff the set $\{k\}$ is admissible.

Proof. Assume first that $k^{(1)} \neq 0$ and at least one of the numbers $r_{m}$ is odd. Then it is obvious that the masses $M_{k^{(m)}-1}^{(K)}$ and $M_{k^{(m)}+r_{m}}^{(K)}$ have different signs and hence the weight function cannot be positive. Thus the condition of the proposition is necessary. The sufficiency of the condition is obvious because then all masses $M_{k}^{(K)}$ have the same sign. This sign is positive as is seen from the first non-zero mass. If $k^{(1)} \neq 0$ then the first $r_{1}$ masses $M_{k}^{(K)}$ are zero and should be excluded from consideration and we return to the previously considered situation. Hence the proposition is proven.

As a by-product (by the Favard theorem) we get that for the admissible case one obtains OP with the positive recurrence coefficients $u_{n}^{(K)}>0$.

REMARK 1. The proposition is obviously valid if the polynomials $P_{n}(x)$ have only discrete spectrum bounded from the above. Then the "admissible" set $\{k\}$ is defined in analogous manner.

Note that M. G. Krein [16] considered a continual analogue of this problem for a set of the Darboux transformations of the Sturm-Liouville equation (see also [5] for the modern interpretation of this situation for the Schrödinger equation with the "nodeless potentials").

4. General scheme of construction for the Racah polynomials. In this section we show how our scheme works for the case of the Racah polynomials.

The Racah polynomials are defined as [14]

$$
R_{n}(x(s) ; \alpha, \beta, \gamma, \delta)={ }_{4} F_{3}\left(\begin{array}{c}
-n, n+\alpha+\beta+1,-s, s+\gamma+\delta+1 \\
\alpha+1, \beta+\delta+1, \gamma+1
\end{array}\right)
$$

where

$$
x(s)=s(s+\gamma+\delta+1)
$$

and one of three conditions

$$
\alpha+1=-N, \text { or } \beta+\delta+1=-N \text { or } \gamma+1=-N
$$

is fulfilled, where $N$ is a positive integer.

The Racah polynomials satisfy the three-term recurrence relation [14]

$$
\xi_{n} R_{n+1}(x(s))-\left(\xi_{n}+\eta_{n}\right) R_{n}(x(s))+\eta_{n} R_{n-1}(x(s))=x(s) R_{n}(x(s)),
$$

where

$$
\begin{aligned}
\xi_{n} & =\frac{(n+\alpha+\beta+1)(n+\alpha+1)(n+\beta+\delta+1)(n+\gamma+1)}{(2 n+\alpha+\beta+1)(2 n+\alpha+\beta+2)} \\
\eta_{n} & =\frac{n(n+\alpha+\beta-\gamma)(n+\beta)(n+\alpha-\delta)}{(2 n+\alpha+\beta+1)(2 n+\alpha+\beta)}
\end{aligned}
$$

The monic Racah polynomials $P_{n}(x(s))=\sigma_{n} R_{n}(x(s))$ satisfy the recurrence relation (1.1) with

$$
u_{n}=\xi_{n-1} \eta_{n}, b_{n}=-\xi_{n}-\eta_{n} .
$$


The coefficients $\sigma_{n}$ satisfy the relation

$$
\frac{\sigma_{n+1}}{\sigma_{n}}=\xi_{n}
$$

The Racah polynomials are orthogonal with respect to a finite discrete measure: the jumps are located at the points $x(s)$ such that

$$
s=0,1, \cdots, N \text {. }
$$

As is seen from (4.1) the polynomials $R_{n}(x(s))$ possess an important duality property: if $x(s)$ coincides with one of the spectral points (i.e. if $s$ belongs to the set (4.8)) then $R_{n}(x(s))$ are also polynomials of $s$-th order in the argument $\mu_{n}=$ $n(n+\alpha+\beta+1)$. This property is crucial for the further construction.

Consider $K$ Christoffel transforms with

$$
\alpha_{k}=j_{k}\left(j_{k}+\gamma+\delta+1\right), k=1,2, \cdots, K<N
$$

where $j_{k}$ are arbitrary chosen integers among the set $0,1, \cdots, N$. Then from (2.4) we derive the following formulas

$$
\begin{aligned}
& u_{n}^{(K)}=u_{n} \frac{\xi_{n+K-1}}{\xi_{n-1}} \frac{\mathcal{P}(n+1 ; K) \mathcal{P}(n-1 ; K)}{\mathcal{P}^{2}(n ; K)} \\
& b_{n}^{(K)}=b_{n+K}+\xi_{n+K} \frac{\tilde{\mathcal{P}}(n+1 ; K)}{\mathcal{P}(n+1 ; K)}-\xi_{n+K-1} \frac{\tilde{\mathcal{P}}(n ; K)}{\mathcal{P}(n ; K)}
\end{aligned}
$$

where

$$
\begin{aligned}
\mathcal{P}(n ; K) & =\left|\begin{array}{cccc}
R_{n+K-1}\left(\alpha_{1}\right) & R_{n+K-2}\left(\alpha_{1}\right) & \cdots & R_{n}\left(\alpha_{1}\right) \\
R_{n+K-1}\left(\alpha_{2}\right) & R_{n+K-2}\left(\alpha_{2}\right) & \cdots & R_{n}\left(\alpha_{2}\right) \\
\cdots & \cdots & \cdots & \cdots \\
R_{n+K-1}\left(\alpha_{K}\right) & R_{n+K-2}\left(\alpha_{K}\right) & \cdots & R_{n}\left(\alpha_{K}\right)
\end{array}\right|, \\
\tilde{\mathcal{P}}(n ; K) & =\left|\begin{array}{cccc}
R_{n+K}\left(\alpha_{1}\right) & R_{n+K-2}\left(\alpha_{1}\right) & \cdots & R_{n}\left(\alpha_{1}\right) \\
R_{n+K}\left(\alpha_{2}\right) & R_{n+K-2}\left(\alpha_{2}\right) & \cdots & R_{n}\left(\alpha_{2}\right) \\
\cdots & \cdots & \cdots & \cdots \\
R_{n+K}\left(\alpha_{K}\right) & R_{n+K-2}\left(\alpha_{K}\right) & \cdots & R_{n}\left(\alpha_{K}\right)
\end{array}\right| .
\end{aligned}
$$

From the duality property of $R_{n}\left(\alpha_{k}\right)$ we see that the transformed coefficients $u_{n}^{(K)}, b_{n}^{(K)}$ are rational functions of the argument $n$. Hence the transformed polynomials $R_{n}^{(K)}(x)$ belong to GPC.

We thus get

Proposition 2. If the parameters $\alpha_{i}, i=1,2, \cdots, K$ satisfy the "admissible" conditions, then OP obtained from the Racah polynomials after $K$ Christoffel transformations at the points $\alpha_{i}$ belong to GPC and have the positive weight function.

Clearly, this statement is valid for specializations of the Racah polynomials (Hahn, Meixner, Krawtchouk, etc.): OP obtained from these polynomials by applying of $K$ Christoffel transforms with admissible parameters $\alpha_{k}$ satisfy needed conditions for GPC: their recurrence coefficients are rational of the argument $n$ whereas their weight functions are positive and explicitly known. One can obtain infinitely many different 
OP belonging to GPC. All such OP can be treated as "closest" to the "classical" OP of a discrete variable in the following sense.

As was shown in [25] all OP obtained from the "classical" OP (such as AskeyWilson, Racah, etc.) by a finite number of linear spectral transforms (i.e. CT and GT) belong to the class of semi-classical OP (SCOP) in a sense of definition [19]. SCOP possess many useful properties similar to those of the classical OP (in particular, the discrete masses $M_{k}$ (if they exist) satisfy the linear first-order recurrence equation (see, e.g. [19], [25]). However the recurrence coefficients for the generic semi-classical polynomials cannot be expressed in terms of the elementary functions; they are rather discrete analogies of the Painlevé transcendents [20], [25]. Hence the polynomials obtained by the our procedure from the "classical" OP form a subclass of SCOP with the rational recurrence coefficients. In this respect these polynomials are "more close" to the classical OP than the generic semi-classical OP.

Note that, in contrast to $\mathrm{CT}$, the Geronimus transform does not preserve this property for the Racah polynomials. However, for some special values of the parameters $\alpha_{i}, \chi_{i}$ this property holds. We consider one such special case in the next section.

5. Example: modified Charlier polynomials on "defected" lattice. Consider the simplest example connected with the Charlier polynomials satisfying the recurrence relation (1.1) with the coefficients [14]

$$
u_{n}=a n, b_{n}=a+n,
$$

where $a>0$ is an arbitrary positive parameter. The spectral points of the Charlier polynomials are located at $x=0,1,2, \cdots$. Corresponding masses form the Poisson distribution:

$$
M_{k}=\exp (-a) a^{k} / k !, k=0,1, \cdots .
$$

The explicit expression of these polynomials is [14]

$$
P_{n}(x)=(-a)_{2}^{n} F_{0}\left(\begin{array}{c}
-n,-x \\
-
\end{array}-1 / a\right) .
$$

Perform now $K$ Christoffel transforms choosing the parameters $\alpha_{i}$ from a subset of the set of spectral points $\alpha_{i}=x_{i}, i=1,2, \cdots, K$. Then from the formulas $(2.4)$ and (5.3) we get

$$
\begin{aligned}
& u_{n}^{(N)}=a n \frac{\mathcal{P}(n+1) \mathcal{P}(n-1)}{\mathcal{P}^{2}(n)} \\
& b_{n}^{(N)}=a+n+N-a\left(\frac{\tilde{\mathcal{P}}(n+1)}{\mathcal{P}(n+1)}-\frac{\tilde{\mathcal{P}}(n)}{\mathcal{P}(n)}\right),
\end{aligned}
$$

where $\mathcal{P}(n)$ and $\tilde{\mathcal{P}}(n)$ are defined by the formulas $(4.11)$ with

$$
R_{n}\left(\alpha_{i}\right)={ }_{2} F_{0}\left(\begin{array}{c}
-n,-\alpha_{i} \\
-
\end{array}-1 / a\right) .
$$

Thus $R_{n}\left(\alpha_{i}\right)$ is a polynomial in the argument $n$ of the order $x_{i}$. Consequently, $\mathcal{P}(n)$ and $\tilde{\mathcal{P}}(n)$ are some polynomials in $n$. 
Taking all possible admissible sets $\{k\}$ for the parameters $\alpha_{k}$ we can construct infinitely many modified Charlier polynomials with the positive discrete weight functions. The corresponding masses form the "perturbed" Poisson distribution:

$$
M_{k}=\kappa \frac{\exp (-a) a^{k}}{k !} \prod_{j=1}^{K}\left(k-\alpha_{j}\right), k=0,1, \cdots,
$$

where $\kappa$ is an appropriate normalization constant. In expression (5.6) the discrete masses $M_{k}$ are positive at the infinite number of the points $k=0,1, \cdots$ excepting the points $\alpha_{1}, \alpha_{2}, \cdots, \alpha_{K}$ where $M_{k}=0$.

Note that if one performs the single CT with $\alpha=0$ then one gets the same Charlier polynomials with the shifted argument: $P_{n}^{(1)}(x ; 0)=P_{n}(x-1)$. Hence if one performs $K$ Christoffel transforms with the parameters $\alpha_{k}$ coinciding with the first $K$ levels of the Charlier polynomials $\left(\alpha_{1}=0, \alpha_{2}=1, \cdots, \alpha_{K}=K-1\right)$ then

$$
P^{(K)}(x ; 0,1, \cdots, K-1)=P_{n}(x-K) .
$$

Thus all CT at the first levels are "non-interesting" and in order to get non-trivial examples we should consider CT with the parameters $\alpha_{k}$ such that $\alpha_{1} \neq 0$ (here $\alpha_{1}$ denotes the smallest $\alpha_{k}$ ).

Consider a simple non-trivial example. Assume that $K=2$ and $\alpha_{1}=1, \alpha_{2}=2$. This means that we perform 2 CT removing the spectral points $x=1,2$ from the measure of the Charlier polynomials. This choice is admissible, i.e. the corresponding weight function is positive at the points $x=0,3,4,5,6, \cdots$ Corresponding recurrence coefficients are given by formulas (5.4) with

$$
\begin{aligned}
& \mathcal{P}(n)=\frac{n^{2}+(1-2 a) n+a^{2}}{a^{3}}, \\
& \tilde{\mathcal{P}}(n)=\frac{2\left(n^{2}+2(1-a) n+a^{2}-a\right)}{a^{3}} .
\end{aligned}
$$

It is easily seen from (5.4) and (5.8) that $u_{n}^{(2)}>0, n=1,2, \cdots$ in agreement with the Favard theorem.

The masses

$$
M_{k}=\frac{e^{-a}}{a^{2}-2 a+2} \frac{(k-1)(k-2) a^{k}}{k !}
$$

are located at the points $k=0,3,4,5, \cdots$ These masses are normalized, i.e. $\sum_{k=0}^{\infty} M_{k}$ $=1$.

Note that this example can be considered as a discrete analogue of the potential constructed in [10] (see also [5]) by the Darboux transformations of the Schrödinger equation for the harmonic oscillator.

There is one more possibility to construct "perturbed" OP with positive discrete weight function starting from the Charlier polynomials.

For this goal note that when $x_{k}=-k, k=1,2, \cdots$ the recurrence relation

$$
\phi_{n+1}+a n \phi_{n-1}+(a+n) \phi_{n}=-k \phi_{n}
$$

admits the following (special) elementary solutions

$$
\phi_{n}=(-1)^{n} n ! \pi_{k-1}(n),
$$


where

$$
\pi_{k}(n)={ }_{2} F_{0}\left(\begin{array}{c}
n+1,-k \\
-
\end{array} ; 1 / a\right)
$$

are some polynomials in $n$ of the order $k$. (Actually these polynomials coincide with the Charlier polynomials with $a \rightarrow-a$ as is seen from (5.5)).

Performing one GT with the function $\phi_{n}$ at $\alpha=-J$ we get new polynomials with the rational recurrence coefficients as is seen from (2.10)

$$
\tilde{u}_{n}=a n \frac{\pi_{J-1}(n) \pi_{J-1}(n-2)}{\pi_{J-1}^{2}(n-1)}, \tilde{b}_{n}=a+n-1+\frac{\pi_{J-1}(n)}{\pi_{J-1}(n-1)}-\frac{\pi_{J-1}(n+1)}{\pi_{J-1}(n)}
$$

According to the transformation of the weight function under the Geronimus transform (2.11) we have

$$
\tilde{w}(x)=\tilde{M}_{-J} \delta(x+J)+\sum_{k=0}^{\infty} \tilde{M}_{k} \delta(x-k),
$$

where $M_{J}$ is the mass inserted at the point $x=-J$ and

$$
\tilde{M}_{k}=\kappa \frac{a^{k}}{(k+J) k !}
$$

( $\kappa>0$ is a normalization constant).

Obviously, the weight function $\tilde{w}(x)$ will be positive iff $\tilde{M}_{-J}>0$. In turn, this condition depends on the sign of the parameter $\chi$ entering the linear combination (2.9). Instead of direct determining the sign of $\chi$ we propose an alternative method allowing to decide whether or not the weight function $\tilde{W}(x)$ is positive. Observe firstly that GT with the spectral parameter $x_{1}=-1$ (i.e. $\phi_{n}=(-1)^{n} n$ !) yields the same Charlier polynomials with the shifted argument $\tilde{P}_{n}(x)=P_{n}(x+1)$. Hence after $J$ Geronimus transforms with the functions $\phi_{n}$ at the points $x_{1}=-1, x_{2}=-2, \cdots, x_{J}=-J$ we get $P_{n}^{(J)}(x)=P_{n}(x+J)$. Then perform $J-1$ Christoffel transforms at the points $x_{1}=-1, x_{2}=-2, \cdots x_{J-1}=1-J$. After this procedure we get the polynomials which are obtained from the Charlier polynomials by the Geronimus transform (5.13) at the point $x_{J}=-J$ (because composition of $\mathrm{CT}$ and GT at the same point is equivalent to identical transformation). On the other hand, this is equivalent to performing of $J-1$ Christoffel transforms with respect to the initial Charlier polynomials at the spectral points $x_{k}=1,2, \cdots, J-1$. Thus the transformed weight function (5.14) is positive iff $J$ is odd.

In particular, taking $J=3$ we return to the above considered case of two CT at the points $x=1,2$.

Thus, for the Charlier polynomials performing of the single GT with the function (5.11) at the point $\alpha=-J$ is equivalent to performing of $J-1 \mathrm{CT}$ at the points $\alpha_{k}=1,2, \cdots, J-1$. More exactly:

$$
\mathcal{G}(0)\left\{P_{n}(x-J)\right\}=\mathcal{C}(1,2, \cdots, J-1)\left\{P_{n}(x)\right\}
$$

or, equivalently,

$$
P_{n}(x-J)=\mathcal{C}(0,1,2, \cdots, J-1)\left\{P_{n}(x)\right\} .
$$


Relation (5.17) coincides with relation (5.7).

Relations like (5.17) (or (5.16)) are special cases of more general self-similar closure conditions for the chain of spectral transformations of OP. Generic solutions of such conditions lead to the so-called semi-classical polynomials on the uniform grid (for details see [25]).

6. Duality properties. Return to the polynomials $\tilde{P}_{n}(x)$ obtained in the previous section with $K=2$ and $\alpha_{1}=1, \alpha_{2}=2$. These polynomials can be written in the form

$$
\tilde{P}_{n}(x)=\frac{q_{n-1} P_{n+2}(x)+r_{n} P_{n+1}(x)+a^{2} q_{n} P_{n}(x)}{q_{n-1}(x-1)(x-2)}, n=0,1,2, \cdots,
$$

where $P_{n}(x)$ are the standard (monic) Charlier polynomials (5.3) and

$$
q_{n-1}=n^{2}+(1-2 a) n+a^{2}, r_{n}=2 a\left(n^{2}+2 n(1-a)+a^{2}-a\right) .
$$

Alternatively, (from (5.16)) these polynomials can be obtained by the single Geronimus transform:

$$
\tilde{P}_{n}(x+3)=P_{n}(x)+\frac{n q_{n}}{q_{n-1}} P_{n-1}(x)
$$

The polynomials $\tilde{P}_{n}(x)$ defined by (6.1) (or (6.3)) can be presented in a concise form in terms of hypergeometric function

$$
\tilde{P}_{n}(x)=\frac{(-1)^{n} a^{n-1}\left(a q_{n-1}-n q_{n}\right)}{q_{n-1}}{ }_{3} F_{1}\left(\begin{array}{c}
-n,-x+3,1+g_{n} \\
g_{n}
\end{array} \mid-a^{-1}\right),
$$

where

$$
g_{n}=-n+\frac{a q_{n-1}}{q_{n}}
$$

The polynomials $\tilde{P}_{n}(x)$ are orthogonal on the "defected" uniform grid $x=0,3,4,5$, $\cdots$. The orthogonality relation reads

$$
\sum_{k=0}^{\infty} \tilde{w}_{k} \tilde{P}_{n}(k) \tilde{P}_{m}(k)=\tilde{h}_{n} \delta_{n m}
$$

where

$$
\begin{aligned}
\tilde{w}_{k} & =\frac{e^{-a}}{a^{2}-2 a+2} \frac{(k-1)(k-2) a^{k}}{k !}, \\
\tilde{h}_{n} & =\frac{a^{2}}{a^{2}-2 a+2} a^{n} n ! \frac{q_{n}}{q_{n-1}} .
\end{aligned}
$$

The Charlier polynomials $P_{n}(x)$ form a complete system. Clearly, the polynomials $\tilde{P}_{n}(x)$ form a complete system as well (because the moment problem for these polynomials is determinate [1]). Hence we have the dual orthogonal relation [4]

$$
\sum_{n=0}^{\infty} \frac{\tilde{P}_{n}(k) \tilde{P}_{n}\left(k^{\prime}\right)}{\tilde{h}_{n}}=\frac{\delta_{k k^{\prime}}}{\tilde{w}_{k}}
$$


where $k, k^{\prime}=0,3,4,5, \cdots$.

For $k=0,3,4,5, \cdots$ it is convenient to write down the polynomials $\tilde{P}_{n}(k)$ in the form

$$
\tilde{P}_{n}(k)=\frac{(-a)^{n-k+2}}{q_{n-1}} Q_{k}(n) .
$$

It is easily seen from (6.4) that $Q_{k}(n)$ are monic polynomials of $k$-th order in the argument $n$, namely

$$
Q_{0}=1 \text {, }
$$

$$
Q_{k}(n)=(-1)^{k} a^{k-3}\left(a q_{n-1}-n q_{n}\right)_{3} F_{1}\left(\begin{array}{c}
-n,-k+3,1+g_{n} \\
g_{n}
\end{array} \mid-a^{-1}\right), k=3,4,5, \cdots \text {. }
$$

The first 4 polynomials are

$$
\begin{aligned}
Q_{0} & =1 \\
Q_{3}(n) & =n^{3}+3(1-a) n^{2}+\left(3 a^{2}-3 a+2\right) n-a^{3} \\
Q_{4}(n) & =n^{4}+2(1-2 a) n^{3}+\left(6 a^{2}-4 a-1\right) n^{2}+2\left(a^{2}-2 a^{3}-1\right) n+a^{4} \\
Q_{5}(n) & =n^{5}-5 a n^{4}+5\left(2 a^{2}-1\right) n^{3}+5 a\left(1-2 a^{2}\right) n^{2}+\left(5 a^{4}+4\right) n-a^{5} .
\end{aligned}
$$

From (6.9) we get that the polynomials $Q_{k}(n)$ are orthogonal on the uniform grid

$$
\sum_{n=0}^{\infty} \rho_{n} Q_{k}(n) Q_{k^{\prime}}(n)=\frac{k ! a^{k}}{(k-1)(k-2)} \delta_{k k^{\prime}}
$$

with the weight function

$$
\rho_{n}=\frac{e^{-a} a^{n+2}}{n ! q_{n-1} q_{n}}
$$

Thus the polynomials $Q_{n}(k)$ can be considered as orthogonal polynomials but with absent $Q_{1}(n)$ and $Q_{2}(n)$. Hence these polynomials are not the ordinary OP in a sense of [8] (recall that all the orders $k=0,1,2, \cdots$ should be presented for the ordinary OP). Nevertheless the polynomials $Q_{k}(n)$ possess many properties similar to those of the ordinary OP. The most important from them is completeness of the system $Q_{k}(n)$. This means that any sequence $\phi_{n}$ such that $\sum_{n=0}^{\infty} \rho_{n}\left|\phi_{n}\right|^{2}<\infty$ can be uniquely expanded in terms of the polynomials $Q_{k}(n)$

$$
\phi_{n}=\sum_{k=0,3,4, \cdots} t_{k} Q_{k}(n)
$$

where the coefficients $t_{k}$ obey the Parseval identity

$$
\sum_{k=0,3,4, \cdots}|t|_{k}^{2}=\sum_{n=0}^{\infty} \rho_{n}\left|\phi_{n}\right|^{2}
$$

The completeness property and the Parseval identity follow from general results concerning orthogonal polynomials having only a discrete measure consisting from a numerable number of jumps (see, e.g. [4, Theorem 5.3.3]). 
The polynomials $Q_{k}(x)$ satisfy some linear difference second-order Sturm-Liouville equation in argument $x$. In order to find this equation we start with the recurrence relation for the polynomials $\tilde{P}_{n}(x)$

$$
\tilde{P}_{n+1}(x)+\tilde{u}_{n} \tilde{P}_{n-1}(x)+\tilde{b}_{n} \tilde{P}_{n}(x)=x \tilde{P}_{n}(x),
$$

where

$$
\begin{aligned}
& \tilde{u}_{n}=a n \frac{q_{n} q_{n-2}}{q_{n-1}^{2}}, \\
& \tilde{b}_{n}=a+n+2-\left(\frac{r_{n+1}}{q_{n}}-\frac{r_{n}}{q_{n-1}}\right) .
\end{aligned}
$$

Then using definition (6.10) we arrive at the difference equation for the polynomials $Q_{k}(x)$

$$
L Q_{k}(x)=k Q_{k}(x), \quad k=0,3,4,5, \cdots,
$$

where $L$ is a second-order difference operator acting on the argument $x$ and defined as

$$
L=-a \frac{q_{x-1}}{q_{x}} \Delta+\frac{x q_{x}}{q_{x-1}} \nabla
$$

$(\Delta, \nabla$ are the standard difference derivative operators defined on the space of functions $\psi(x)$ by $\Delta \psi(x)=\psi(x+1)-\psi(x), \nabla \psi(x)=\psi(x)-\psi(x-1))$. It is interesting to compare the operator $L$ with the corresponding operators for the classical OP on uniform grids (i.e. for the Charlier, Krawtchouk, Meixner and Hahn polynomials). These operators have the expression [22]

$$
L=-(\sigma(x)+\tau(x)) \Delta+\sigma(x) \nabla,
$$

where $\sigma(x)$ is a polynomial of degree $\leq 2$ and $\tau(x)$ is a linear function of $x$. The operator (6.21) possesses a remarkable property: it sends any polynomial $p(x)$ to another polynomial of the same degree (for theory of operators with this property see [19]).

Our operator (6.20) has more complicated properties. Obviously $L\{1\}=0$, but generally for any polynomial $p(x)$ of arbitrary degree $n$ we have $L\{p(x)\}=\frac{\tilde{p}(x)}{q_{x} q_{x-1}}$, where $\tilde{p}(x)$ is a polynomial of degree $\leq n+4$. Thus the operator (6.20) sends a set of polynomials to a set of rational functions. Only if $p(x)$ coincides with some of the polynomials $Q_{n}(x), n=3,4,5, \cdots$ we get the eigenvalue property (6.19). Note that the operator $L$ defined by (6.20) has no polynomial eigenvalue solutions if the degree of the polynomials is 1 or 2 .

7. Generating functions. We already noted that the generating function of OP belonging to GPC satisfies a linear differential equation of. However for the case of OP obtained from classical OP (of a discrete variable) by a finite number of spectral transformations it is possible to construct generating functions explicitly without solving this differential equation.

In this section we find two generating functions for the polynomials $\tilde{P}_{n}(x)$ and $Q_{k}(n)$ constructed in the previous section. 
Introduce the generating function for the standard Charlier polynomials [14]

$$
\Phi(t, x)=e^{-a t}(1+t)^{x}=\sum_{n=0}^{\infty} \frac{P_{n}(x) t^{n}}{n !} .
$$

In order to construct the generating function for the polynomials $\tilde{P}_{n}(x)$ we take the representation (6.3) and get the relation

$$
\sum_{n=0}^{\infty} \frac{q_{n-1} t^{n}}{n !} \tilde{P}_{n}(x+3)=\sum_{n=0}^{\infty} \frac{q_{n-1} t^{n}}{n !} P_{n}(x)+t \sum_{n=0}^{\infty} \frac{q_{n+1} t^{n}}{n !} P_{n}(x) .
$$

The problem is thus reduced to calculating of two sums in the rhs of (7.2). To do this we note that

$$
\begin{aligned}
& \sum_{n=0}^{\infty} \frac{n P_{n}(x) t^{n}}{n !}=t \partial_{t} \Phi(t, x) \\
& \sum_{n=0}^{\infty} \frac{n(n-1) P_{n}(x) t^{n}}{n !}=t^{2} \partial_{t}^{2} \Phi(t, x)
\end{aligned}
$$

Using these identities we can easily calculate both sums in rhs (7.2). Omitting the technical details we present the result: the generating functions $\tilde{\Phi}(t, x)$ for the polynomials $P_{n}(x)$ is

$$
\tilde{\Phi}(t, x)=\phi(t, x) e^{-a t}(1+t)^{x-4}=\sum_{n=0}^{\infty} \frac{q_{n-1} t^{n}}{n !} \tilde{P}_{n}(x),
$$

where $\phi(t, x)$ is the polynomial in $t$ and $x$ :

$$
\phi(t, x)=a^{2} t^{4}+2 a(2 a-x) t^{3}+\left(x^{2}-(4 a+1) x+6 a^{2}\right) t^{2}+2\left((1-a) x+2 a^{2}\right) t+a^{2} .
$$

The generating function for the dual polynomials can be found analogously. We start from the generating function for the standard Charlier polynomials

$$
(z-a)^{n} e^{z}=\sum_{x=0}^{\infty} \frac{P_{n}(x) z^{x}}{x !} .
$$

Taking then the representation (6.1) and using (7.5) we get

$$
\begin{aligned}
& \sum_{x=0}^{\infty} \frac{(x-1)(x-2) z^{x} \tilde{P}_{n}(x)}{x !}= \\
& e^{z}\left((z-a)^{n+2}+\frac{r_{n}}{q_{n-1}}(z-a)^{n+1}+\frac{a^{2} q_{n}}{q_{n-1}}(z-a)^{n}\right) .
\end{aligned}
$$

Now from (6.10) we get finally the generating function for the polynomials $Q_{n}(x)$

$$
\sum_{k=0}^{\infty} \frac{(k-1)(k-2)(-z / a)^{k} Q_{k}(x)}{k !}=e^{z}(z-a)^{x}(-a)^{-x-2} \psi(x, z),
$$

where $\psi(x, z)$ is the polynomial in $x$ and $z$ :

$$
\psi(x, z)=q_{x-1}(z-a)^{2}+r_{x}(z-a)+a^{2} q_{x} .
$$


8. Algebraic scheme: connection with factorization chain. In this section we consider relation of the obtained OP with the so-called discrete factorization chain.

Recall basic definitions and relations connected with the discrete factorization chain (see, e.g. [27]).

Consider a set of (monic) orthogonal polynomials $P_{n}(x ; t)$ depending on an additional discrete parameter $t=0,1,2, \cdots$ and satisfying the recurrence relations

$$
\begin{aligned}
& P_{0}(x ; t)=1, P_{1}(x ; t)=x-b_{0}(t), \\
& P_{n+1}(x ; t)+b_{n}(t) P_{n}(x ; t)+u_{n}(t) P_{n-1}(x ; t)=x P_{n}(x ; t), \quad t=0,1,2, \cdots .
\end{aligned}
$$

Let $\alpha(t), t=0,1,2, \cdots$ be a set of parameters such that the following relations between the polynomials hold

$$
P_{n}(x ; t+1)=\frac{P_{n+1}(x ; t)-A_{n}(t) P_{n}(x ; t)}{x-\alpha(t)},
$$

where

$$
A_{n}(t)=\frac{P_{n+1}(\alpha(t) ; t)}{P_{n}(\alpha(t) ; t)}
$$

Comparing (8.2) with (2.1) we see that the polynomials $P_{n}(x ; t+1)$ are obtained from $P_{n}(x ; t)$ by means of the Christoffel transform with the spectral parameter $\alpha(t)$.

The reciprocal transformation from $P_{n}(x ; t+1)$ to $P_{n}(x ; t)$ is given by the Geronimus transformation

$$
P_{n}(x ; t)=P_{n}(x ; t+1)-B_{n}(t) P_{n-1}(x ; t+1),
$$

where the coefficients $B_{n}(t)$ can be presented in the form (2.8).

It is useful to inverse the problem and consider the set of the coefficients $A_{n}(t)$ and $E_{n}(t)$ as independent parameters.

Then we obtain connections between these coefficients and the recurrence coefficients

$$
\begin{aligned}
u_{n}(t) & =A_{n-1}(t) B_{n}(t), n=1,2, \cdots \\
b_{0}(t) & =\alpha(t)-A_{0}(t), b_{n}(t)=\alpha(t)-A_{n}(t)-B_{n}(t), n=1,2, \cdots .
\end{aligned}
$$

Nloreover, there are two basic relations between the coefficients $A_{n}(t), B_{n}(t)$ themselves:

$$
A_{n}(t+1) B_{n+1}(t+1)=A_{n+1}(t) B_{n+1}(t), \quad n=0,1,2, \cdots
$$

and

$$
\begin{aligned}
& A_{0}(t+1)-\alpha(t+1)=A_{0}(t)+B_{1}(t)-\alpha(t), \\
& A_{n}(t+1)+B_{n}(t+1)-\alpha(t+1)=A_{n}(t)+B_{n+1}(t)-\alpha(t), \quad n=1,2, \cdots .
\end{aligned}
$$

The set of equations (8.7), (8.8) is called the discrete factorization chain [27].

Starting from the given solution $A_{n}(t), B_{n}(t)$ of the discrete factorization chain DFC (8.7), (8.8) we can reconstruct a family $P_{n}(x ; t)$ of OP depending on the additional discrete parameter $t$ and related with one another by the Christoffel and Geronimus transforms (see, e.g. [28] where the most general "classical" OP (i.e. the 
Askey-Wilson and the Askey-Ismail OP) are reconstructed from self-similar solutions of the DFC).

REMARK 2. Note that the apparent asymmetry between the first $(n=0)$ and other relations in (8.8) can be removed if one formally puts

$$
B_{0}(t)=0 \text {. }
$$

Note also that the DFC is equivalent to the so-called extended g-algorithm proposed by F.Bauer [6] in theory of numerical approximations (for details concerning this equivalence see, e.g. [28] and [34]).

Following [24], [25], consider periodic closure conditions for the corresponding coefficients (the idea of introducing of such conditions in the case of the Sturm-Liouville equation is due to Veselov and Shabat [31])

$$
A_{n}(t+N)=A_{n}(t), B_{n}(t+N)=B_{n}(t) .
$$

The polynomials (6.4) provide an example of $N=3$ quasiperiodic closure. Indeed, consider the following expressions

$$
\begin{aligned}
& A_{n}(0)=-\frac{a(n+1-a)}{n-a}, B_{n}(0)=-\frac{n(n-a-1)}{n-a}, \alpha(0)=1 ; \\
& A_{n}(1)=-\frac{a(n-a) q_{n}}{(n+1-a) q_{n-1}}, B_{n}(1)=-\frac{n(n-a+1) q_{n-2}}{(n-a) q_{n-1}}, \alpha(1)=2 ; \\
& A_{n}(2)=-\frac{a q_{n-1}}{q_{n}}, B_{n}(2)=-\frac{n q_{n}}{q_{n-1}}, \alpha(2)=0
\end{aligned}
$$

and define other coefficients (for $t>2$ ) by the periodicity $A_{n}(t+3)=A_{n}(t), B_{n}(t+$ $3)=B_{n}(t)$.

It is easily verified that such defined coefficients $A_{n}(t), B_{n}(t)$ do satisfy the relations (8.7), (8.8) with quasiperiodic conditions (8.10). In our case $\sigma=3$. Note that $B_{0}(t)=0$ in accordance with (8.9). This solution corresponds to the Jacobi matrices $H(0), H(1), H(2)$. The matrix $H(0)$ corresponds to the ordinary Charlier polynomials $P_{n}(x ; 0)$ with the recurrence coefficients $u_{n}=a n, b_{n}=a+n$. The matrix $H(1)$ corresponds to the polynomials obtained from the ordinary Charlier polynomials by CT on the level $\alpha(0)=1$. These polynomials $P_{n}(x ; 1)$ have the recurrence coefficients

$$
\begin{aligned}
& u_{n}(1)=a n \frac{(n+1-a)(n-1-a)}{(n-a)^{2}}, \\
& b_{n}(1)=a+n+1-\frac{a(n+2-a)}{n+1-a}+\frac{a(n+1-a)}{n-a} .
\end{aligned}
$$

It is seen that the coefficient $u_{n}(1)<0$ for $n=[a]+1$, where $[a]$ denotes the integer part of the number. Hence the polynomials $P_{n}(x ; 1)$ do not possess a positive weight function. This is expected because the polynomials $P_{n}(x ; 1)$ are obtained from $P_{n}(x ; 0)$ by the single CT at the point $\alpha(0)=1$ which is inside the spectral interval.

Finally, the polynomials $P_{n}(x ; 2)$ coincide with the polynomials (6.4) because they are obtained from $P_{n}(x ; 0)$ by two CT at the points $\alpha(0)=1, \alpha(1)=2$.

The quasiperiodic condition (8.10) means that

$$
P_{n}(x ; t+N)=P_{n}(x-N ; t) .
$$


As it was shown in [25], the closure condition (8.14) leads to the class of semi-classical polynomials on the uniform grid. Equivalently, the semi-classical polynomials on the uniform grids are characterized by quasi-periodic solutions of the factorization chain (8.7), (8.8).

9. Conclusion. We showed how to construct infinitely many orthogonal polynomials with the properties:

(i) the recurrence coefficients $u_{n}$ and $b_{n}$ are rational functions of the argument $n$;

(ii) the weight function is positive and known explicitly;

(iii) these polynomials are expressed in terms of a finite linear combination of hypergeometric functions.

These polynomials belong to a wide class of the so-called semi-classical polynomials of a discrete variable [19], [33]. Nevertheless, polynomials constructed in this paper can be considered as "intermediate" between the "true" classical and the "true" semi-classical OP. Indeed, it is known that for generic semi-classical polynomials the recurrence coefficients $u_{n}, b_{n}$ have rather complicated form and cannot be expressed in terms of elementary functions. In fact, these coefficients are related to the socalled "discrete Painlevé transcendents" [20]. In our case, however, the recurrence coefficients have elementary expression and hence can be considered as degenerated (rational) solutions of the nonlinear discrete Painlevé equations.

Finally note an interesting problem arising in connection with generating function (7.3). There is the well known result by Meixner and Sheffer concerning all possible OP $S_{n}(x)$ arising from the generating function

$$
A(t) e^{x v(t)}=\sum_{n=0}^{\infty} S_{n}(x) t^{n}
$$

where $A(t)$ and $v(t)$ are some functions such that $A(0)=v^{\prime}(0)=1, v(0)=0$. The polynomials $S_{n}(x)$ are called the Sheffer polynomials. It appears (see, e.g. [2]) that the only Sheffer OP are Hermite, Laguerre, Charlier, Meixner and Meixner-Pollaczek polynomials. Al-Salam showed [2] that the condition of this problem can be weakened: consider the generating function

$$
\exp \{F(x, t)\}=\sum_{n=0}^{\infty} S_{n}(x) t^{n}
$$

where $F(x, t)$ is a polynomial in $x$ with coefficients depending in $t$. We then arrive at the same classical Sheffer polynomials.

Now we can formulate the problem:

Find all OP $P_{n}(x)$ generated by the function

$$
F_{1}(x, t) \exp \left\{F_{2}(x, t)\right\}=\sum_{n=0}^{\infty} P_{n}(x) t^{n},
$$

where both $F_{1}(x, t)$ and $F_{2}(x, t)$ are polynomials in $x$ with coefficients depending in $t$. As far as we know this problem was not yet studied. The expression (7.3) provides a non-trivial example of such generating function leading to non-classical OP. In this case $F_{2}(x, t)$ is a first-order polynomial whereas $F_{1}(x, t)$ is a second-order polynomial in $x$. It would be interesting to characterize all such functions (and corresponding OP) satisfying (9.3). 
Acknowledgments. The authors are grateful to B. Samsonov, V. Spiridonov, S. Suslov, A. Veselov and L. Vinet for discussion, to M. E. H. Ismail and A. Magnus for stimulating communications and to a referee for useful remarks and suggestions. The work of A. Zh. was supported in part through funds provided by SCST (Ukraine) Project \#2.4/197, INTAS-96-0700 grant and project 96-01-00281 supported by RFBR (Russia). A.Zh. thanks Centre de recherches mathématiques of the Université de Montréal for hospitality.

\section{REFERENCES}

[1] N. I. Ahieser, Classical Moments Problem and Related Questions in Analysis, Fizmatgiz (in Russian), Moscow, 1961.

[2] W. A. Al-Salam, Characterization theorems for orthogonal polynomials, in Orthogonal Polynomials: Theory and Practice, P. Nevai, ed., NATO ASI Series C: Mathematical and Physical Sciences, 294, Kluwer Academic Publishers, 1990, pp. 1-24.

[3] R. ASKey AND M. E. H. IsMAIL, Recurrence relations, continued fractions and orthogonal polynomials, Mem. Am. Math. Soc. 300, 1984.

[4] F. V. AtKinson, Discrete and Continuous Boundary Problems, Academic Press, NY, 1964.

[5] V. G. BAGRoV AND B. F. SAMSONOV, Darboux transformation, factorization, supersymmetry in one-dimensional quantum mechanics, Teor. i Mat. Fiz., 104 (1995), pp. 356-367.

[6] F. L. BAUER, The g-algorithm, J. Soc. Indust. Appl. Math., 8 (1960), pp. 1-17.

[7] J. A. Charris and M. E. H. Ismail, On sieved orthogonal polynomials. V: Sieved Pollaczek polynomials, SIAM J. Math. Anal., 18 (1987), pp. 1177-1218.

[8] T. ChiHARA, An Introduction to Orthogonal Polynomials, Gordon and Breach, 1978.

[9] T. S. Chinara, Orthogonal polynomials and measures with end point masses, Rocky Mount. J. of Math., 15 (1985), pp. 705-719.

[10] S. Yu. Dubov, V. M. Eleonskit, AND N. E. Kulagin, On the equidistant spectra of anharmonic oscillators, Zh. Eksper. Teor. Fiz., 102 (1992), pp. 814-825.

[11] YA. L. GERONIMUS, On the polynomials orthogonal with respect to a given number sequence, Zap. Mat. Otdel. Khar'kov. Univers. i NII Mat. i Mehan., 17 (1940), pp. 3-18.

[12] Ya. L. Geronimus, On the polynomials orthogonal with respect to a given number sequence and a theorem by W.Hahn, Izv. Akad. Nauk SSSR, 4 (1940), pp. 215-228.

[13] A. Iserles, P. E. Koch, S. P. Nørsett, AND J. M. SANZ-Serna, On polynomials orthogonal with respect to certain Sobolev inner product, J. Approx. Theory, 65 (1991), pp. 151-175.

[14] R. Koekoek AND R. F. SwarTtouw, The Askey scheme of hypergeometric orthogonal polynomials and its q-analogue, Report 94-05, Faculty of Technical Mathematics and Informatics, Delft University of technology, 1994.

[15] T. H. KooRNwinder, Orthogonal polynomials with weight function $(1-x)^{\alpha}(1+x)^{\beta}+M \delta(x+$ 1) $+N \delta(x-1)$, Can. Math. Bull., 27 (1984), pp. 205-214.

[16] M. G. KREIN, On the continual analogues of some Christoffel formula in the theory of orthogonal polynomials, Dokl. AN SSSR, 113 (1957), pp. 970-973.

[17] K. H. KWon, D. W. Lee, AND L. L. LitTlejohn, Differential equations having orthogonal polynomial solutions, J. Comput. Appl. Math., 80 (1997), pp. 1-16.

[18] L. L. LitTLEjohN, On the classification of differential equations having orthogonal polynomials solutions, Anal. Mat., 4 (1984), pp. 35-53.

[19] A. Magnus, Associated Askey-Wilson polynomials as Laguerre-Hahn orthogonal polynomials, in Orthogonal Polynomials and their applications, M. Alfaro et. al., ed., Lect. Notes in Math., 1329, Springer, Berlin, 1988, pp. 261-278.

[20] A. P. Magnus, Painlevé-type differential equations for the recurrence coefficients of semiclassical orthogonal polynomials, J. Comp. Appl. Math., 57 (1995), pp. 215-237.

[21] P. MARoni, Sur la suite de polynômes orthogonaux associée à la forme $u=\delta_{c}+\lambda(x-c)^{-1} L$, Period. Math. Hung., 21:3 (1990), pp. 223-248.

[22] A. F. Nikiforov, S. K. Suslov, and V. B. Uvarov, Classical Orthogonal Polynomials of a Discrete Variable, Springer, 1991.

[23] F. Pollaczek, Sur une généralisation des polynomes de Jacobi, Mémorial des Sciences Mathématique 131, 1956.

[24] V. Spiridonov, L. Vinet, ANd A. Zhedanov, Difference Schrödinger operators with linear and exponential discrete spectra, Lett. Math. Phys., 29 (1993), pp. 63-73.

[25] V. SpIRIDonov, L. Vinet, AND A. ZhedANov, Spectral transformations, self-similar reductions and orthogonal polynomials, J. Phys. A: Math. \& Gen., 30 (1997), pp. 7621-7637. 
[26] V. SPIRIDONOV AND A. ZHEDANOV, Discrete reflectionless potentials, quantum algebras and q-orthogonal polynomials, Ann. Phys., 237 (1995), pp. 126-146.

[27] V. SpIRIdonov And A. Zhedanov, Discrete Darboux transformation, discrete-time Toda lattice and the Askey-Wilson polynomials, Methods and Appl. of Analysis, 2 (1995), pp. 369-398.

[28] V. SpIRIdonov and A. Zhedanov, Discrete-time Volterra chain and classical orthogonal polynomials, J. Phys. A: Math. \& Gen., 30 (1997), pp. 8727-8737.

[29] G. Szegö, Orthogonal Polynomials, AMS, 1959.

[30] G. VALENT, Asymptotic analysis of some associated orthogonal polynomials associated with elliptic functions, SIAM J. Math. Anal., 25 (1994), pp. 749-775.

[31] A. Veselov AND A. Shabat, Dressing chain and spectral theory of Schrödinger operator, Funkt. Anal. i Pril., 27:2 (1993), pp. 1-21.

[32] J. A. WILSON, Some hypergeometric orthogonal polynomials, SIAM J. Math. Anal., 11 (1980), pp. 690-701.

[33] A. Zhedanov, Rational spectral transformations and orthogonal polynomials, J. Comp. Appl. Math., 85 (1997), pp. 67-86.

[34] A. ZHEDANov, On some classes of polynomials orthogonal on arcs of the unit circle connected with symmetric orthogonal polynomials on an interval, J. Approx. Theory, 94 (1998), pp. 73-106. 